Supporting Information

\title{
Significant Enhancement of Energy Storage Performances by Regulating the Dielectric Contrast between Adjacent Layers in the Heterostructural Composites
}

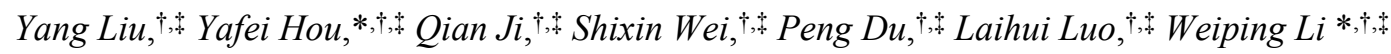

$\dagger$ Department of Microelectronics Science and Engineering, School of Physical Science and Technology, Ningbo University, Ningbo, 315211, P.R. China.

$\$$ Laboratory of Clean Energy Storage and Conversion, Ningbo University, Ningbo, 315211, P.R. China.

Corresponding Author

E-mail addresses: houyafei@nbu.edu.cn (Y. F. Hou)

E-mail addresses: liweiping@nbu.edu.cn (W.P. Li) 

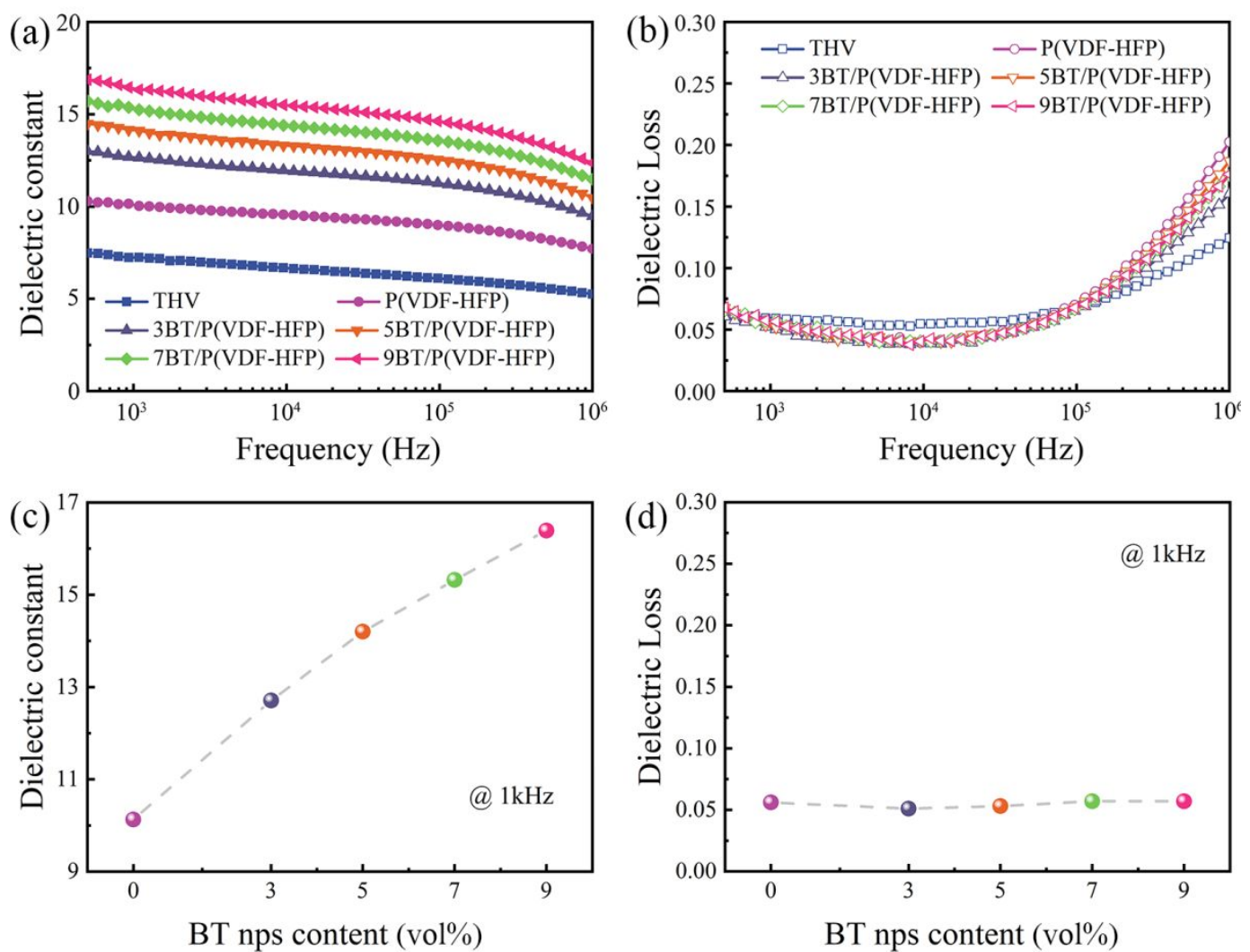

Figure S1 (a) Dielectric constant and (b) dielectric loss of THV and $x \mathrm{BT} / \mathrm{P}(\mathrm{VDF}-\mathrm{HFP})$ as a function of frequency, the variation of (c) dielectric constant and (d) dielectric loss for $x \mathrm{BT} / \mathrm{P}(\mathrm{VDF}-\mathrm{HFP})$ composites with BT nps fraction increase at $1 \mathrm{kHz}$ 

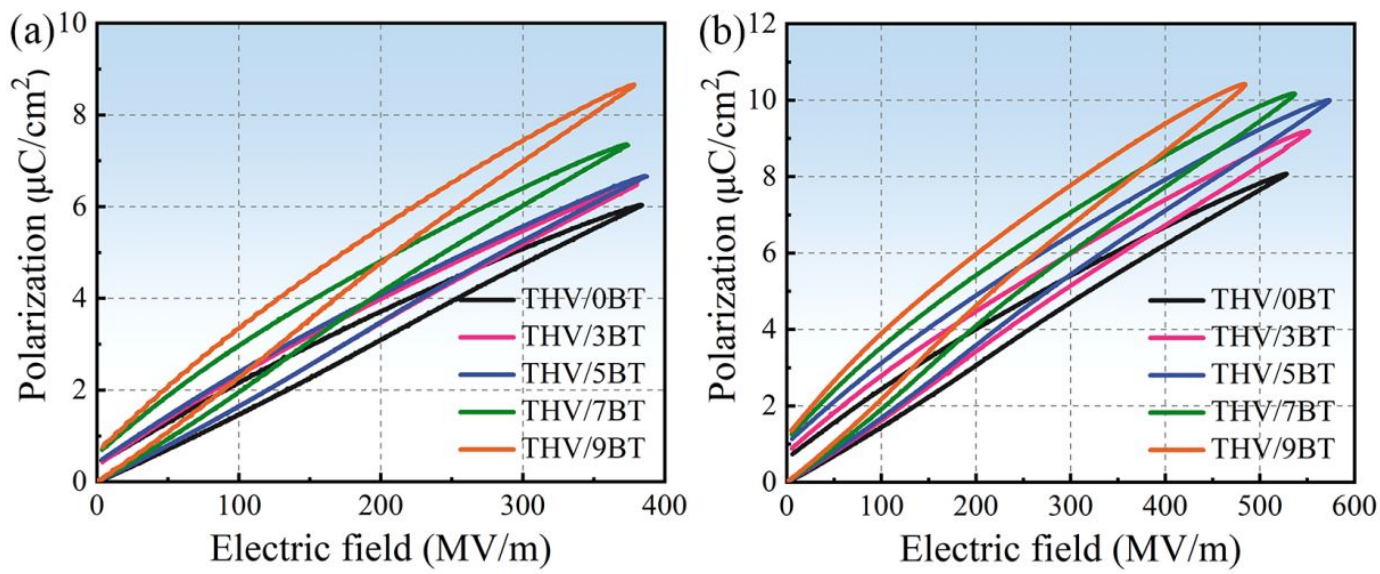

Figure S2 $P-E$ loops of THV $/ x$ BT bilayer composites measured at (a) $380 \mathrm{MV} / \mathrm{m}$, and (b) the corresponding breakdown strength 

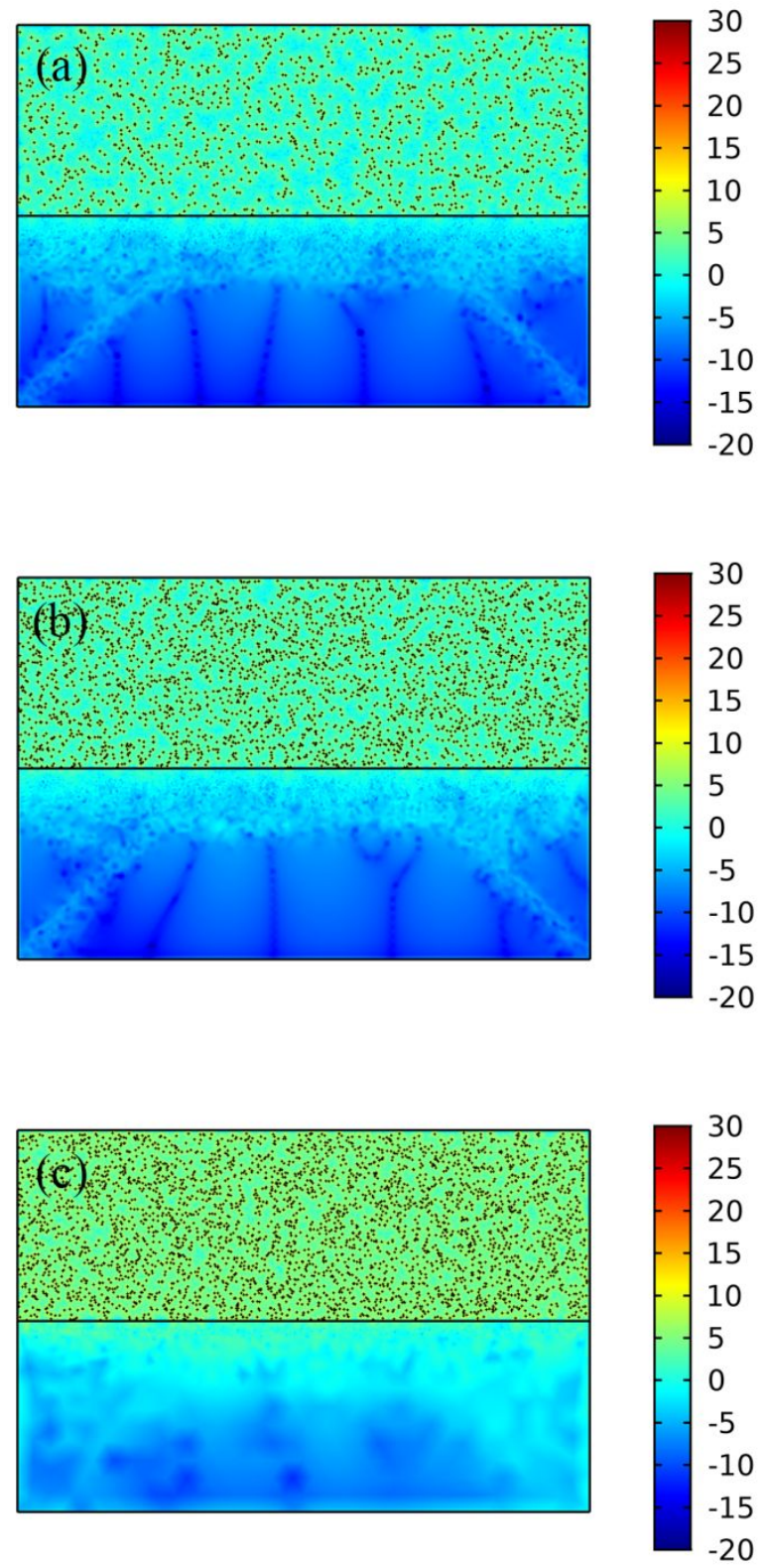

Figure S3 The distribution of space charge density simulated for (a) THV/3BT (b) THV/7BT and (c) THV/9BT bilayer composites. 
Table S1 The statistical results of space charge density in THV layer and entirety film.

\begin{tabular}{cccc}
\hline $\begin{array}{c}\text { Space charge } \\
\text { density }\left(\mathrm{C} / \mathrm{m}^{3}\right)\end{array}$ & THV/3BT & THV/7BT & THV/9BT \\
\hline THV layer & -8.18 & -5.45 & -2.87 \\
Entirety & -2.72 & -0.68 & 1.57 \\
\hline
\end{tabular}




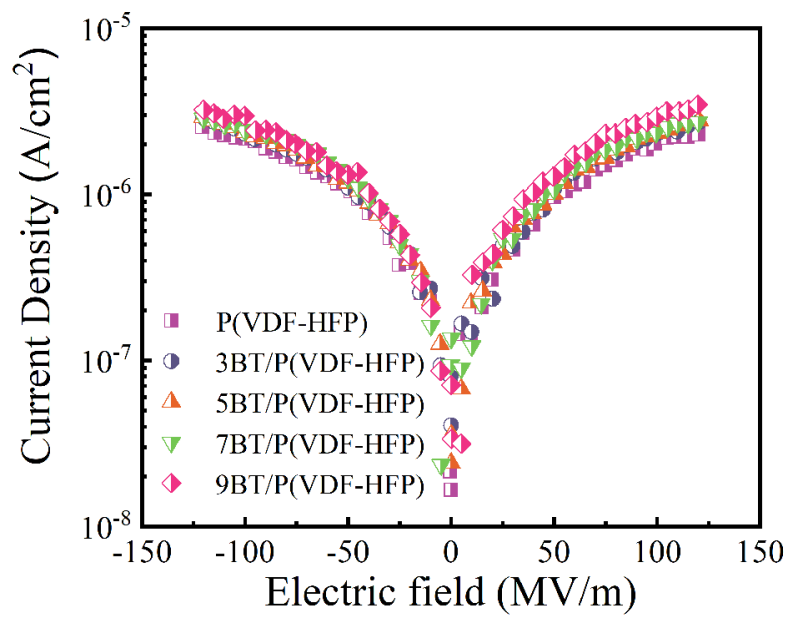

Figure S4 Leakage current density of $x \mathrm{BT} / \mathrm{P}(\mathrm{VDF}-\mathrm{HFP})$ composites 

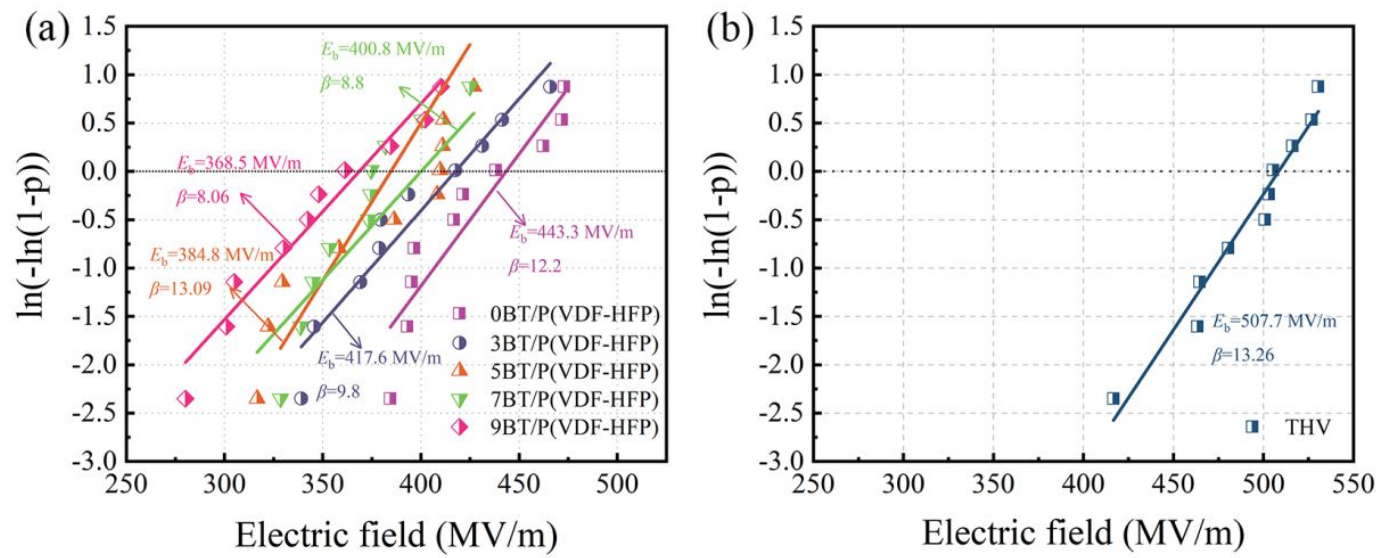

Figure S5 Weibull distribution of (a) $x \mathrm{BT} / \mathrm{P}(\mathrm{VDF}-\mathrm{HFP}$ ) composites and (b) THV film 

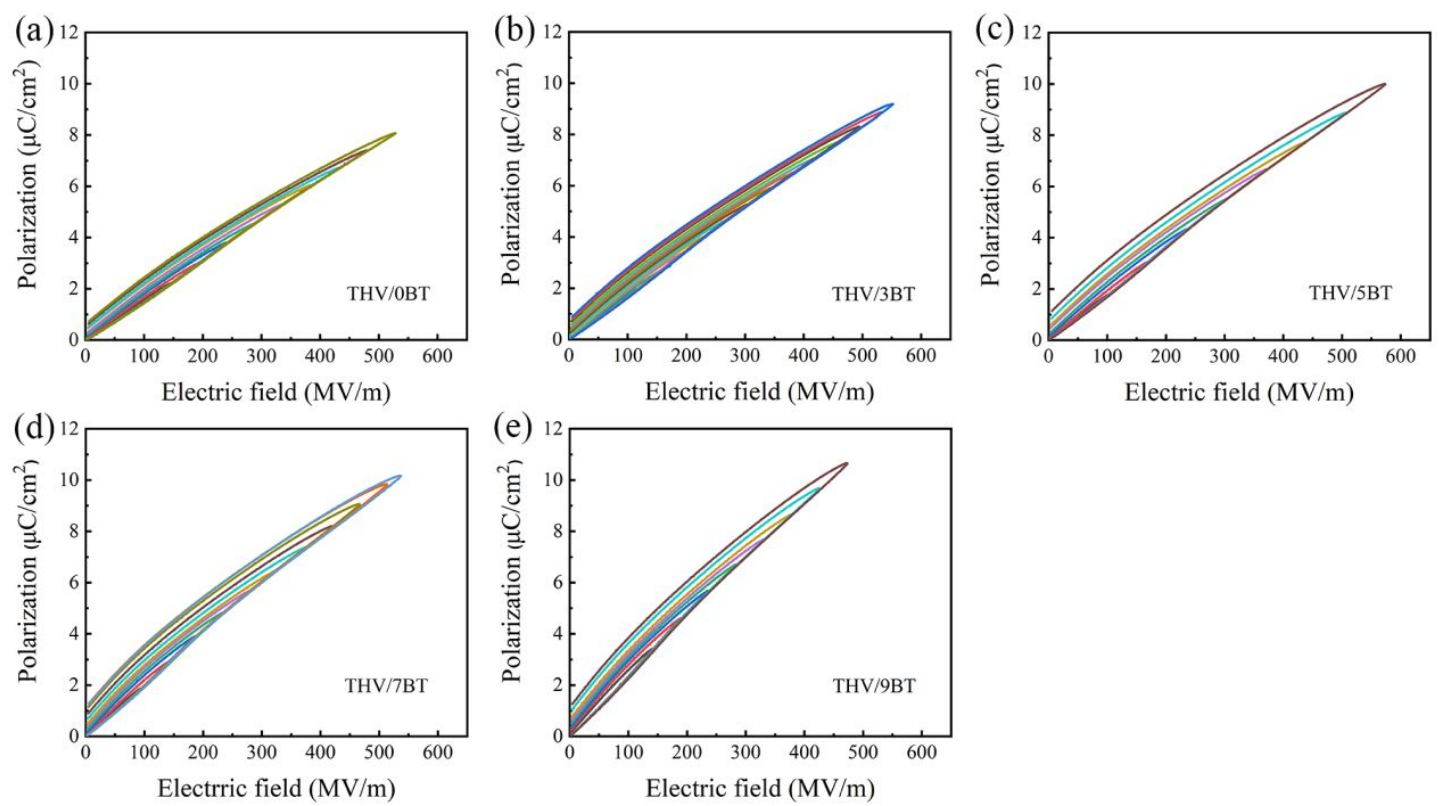

Figure S6 $P$ - $E$ loops of $\mathrm{THV} / x \mathrm{BT}$ composites measured at sequential electric fields 

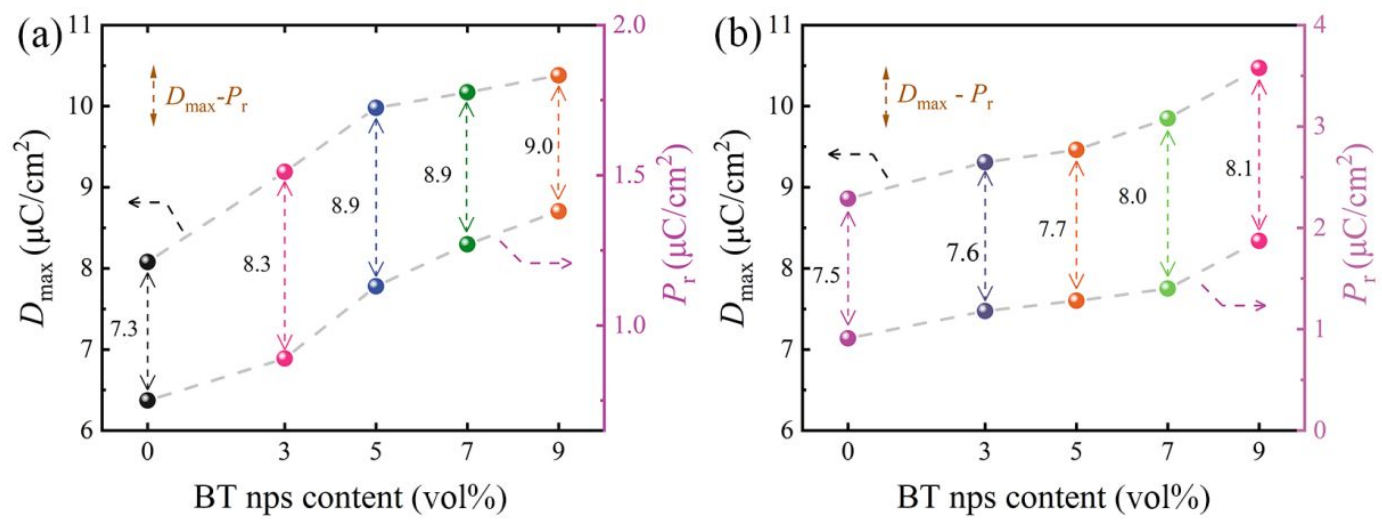

Figure S7 The variations of $D_{\max }, P_{\mathrm{r}}$ and $D_{\max }-P_{\mathrm{r}}$ values for (a) THV/xBT and (b) $\mathrm{xBT} / \mathrm{PVDF}$ composites with BT nps fraction increase at their breakdown strength 

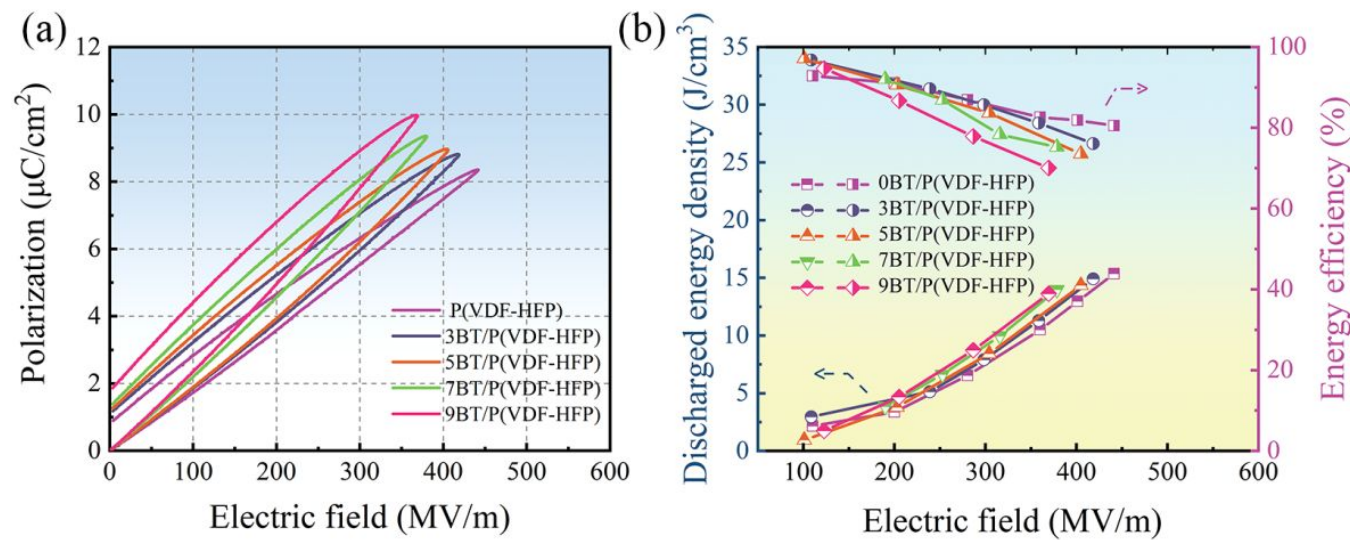

Figure S8 (a) $P-E$ loops of $x \mathrm{BT} / \mathrm{P}(\mathrm{VDF}-\mathrm{HFP})$ composites and (b) the corresponding discharged energy density and energy efficiency of $x \mathrm{BT} / \mathrm{P}(\mathrm{VDF}-\mathrm{HFP})$ composites 


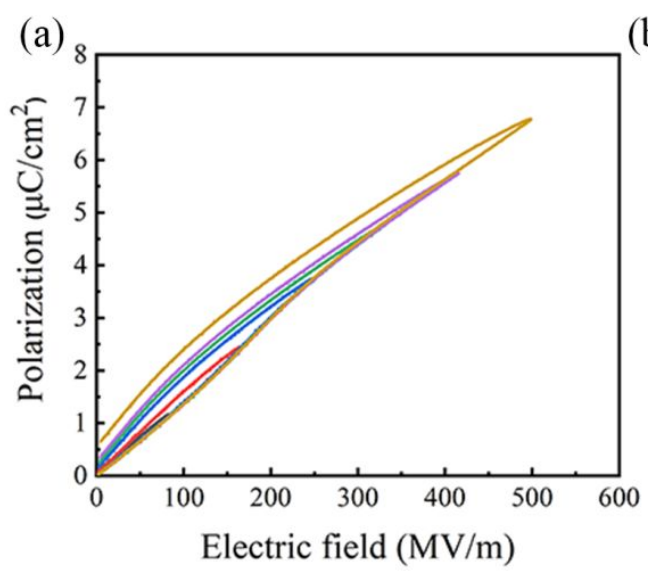

(b)

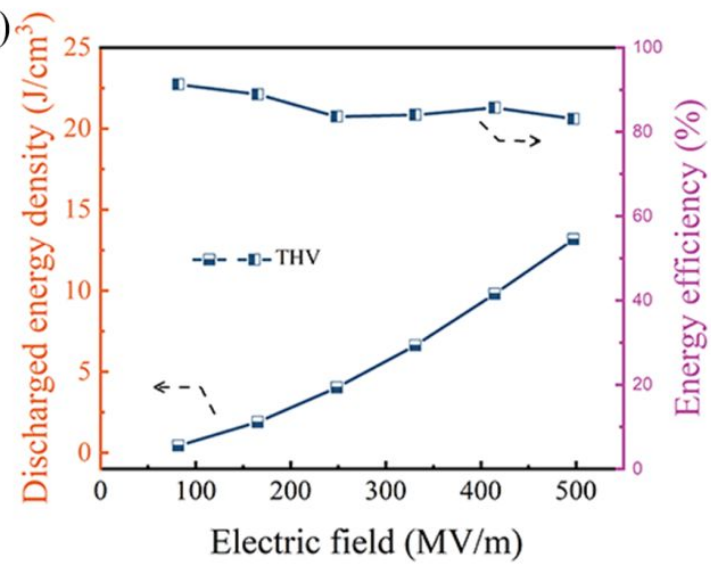

Figure S9 (a) $P-E$ loops of THV film and (b) the corresponding discharged energy density and energy efficiency of THV film 

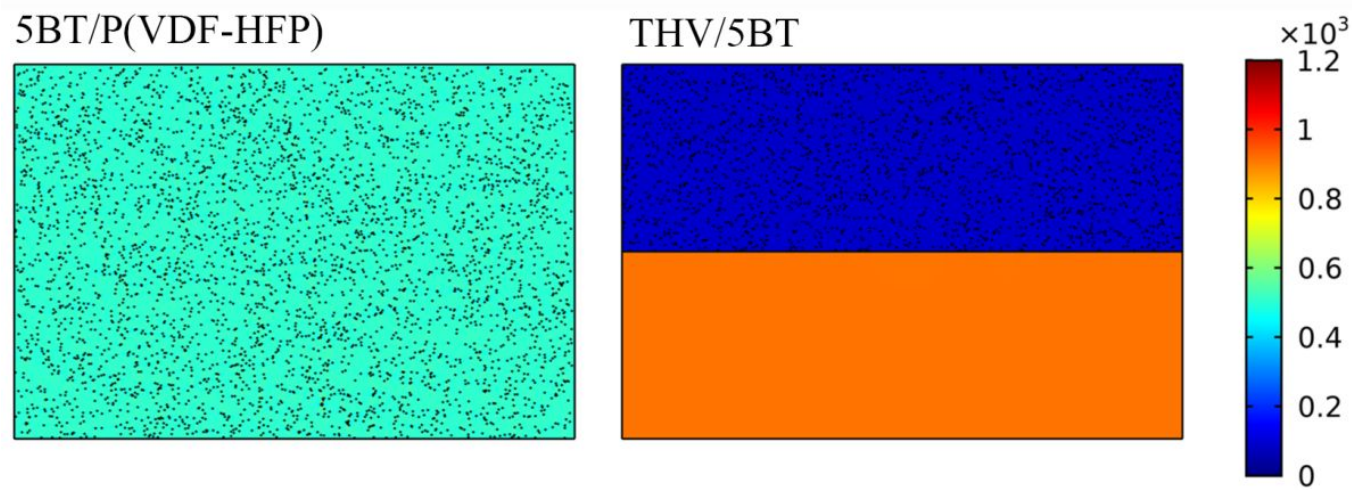

Figure S10 The distribution of electric field simulated for 5BT/P(VDF-HFP) and THV/5BT composites 


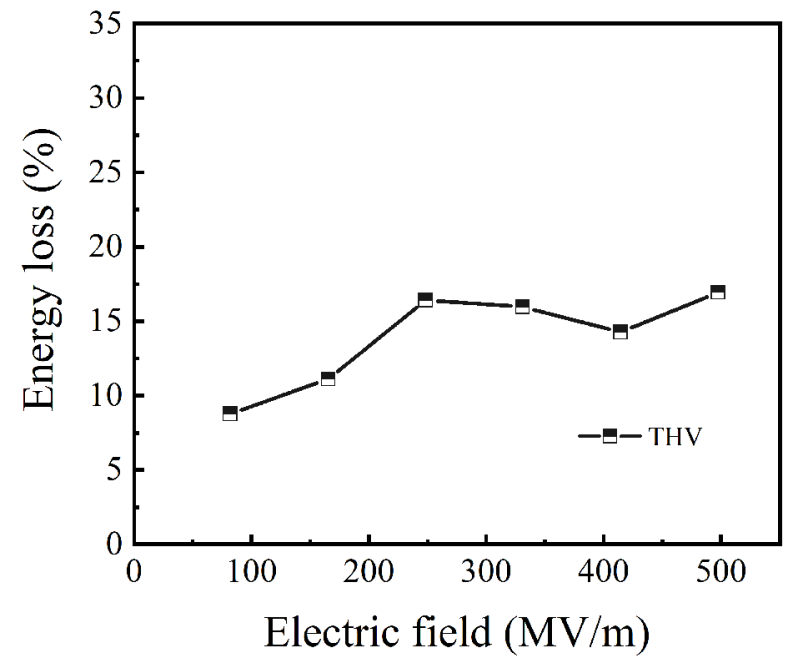

Figure S11 Energy loss $(1-\eta)$ of the THV film 